\title{
Long non-coding RNA signatures as predictors of prognosis in thyroid cancer: a narrative review
}

\author{
Hongyuan Zhao ${ }^{1 \wedge}$, Cristabelle De Souza ${ }^{2,3}$, Vigneshwari Easwar Kumar ${ }^{2}$, Roshni Nambiar ${ }^{2}$, Dake Hao ${ }^{4,5}$, \\ Xiaofeng Zhu ${ }^{6}$, Yi Luo ${ }^{6}$, Shengshan Liu ${ }^{6}$, Lingyun Zhang ${ }^{1}$, Jingqiang Zhu ${ }^{1}$ \\ ${ }^{1}$ Department of Thyroid and Parathyroid Surgery, West China Hospital, Sichuan University, Chengdu, China; ${ }^{2}$ Department of Biochemistry and \\ Molecular Medicine, School of Medicine, University of California, Sacramento, CA, USA; ${ }^{3}$ Institute for Regenerative Medicine and Stem Cell \\ Research, Stanford University, Stanford, CA, USA; ${ }^{4}$ Department of Surgery, School of Medicine, University of California, Sacramento, CA, USA; \\ ${ }^{5}$ Institute for Pediatric Regenerative Medicine, Shriners Hospitals for Children, Sacramento, CA, USA; ${ }^{6}$ Department of Thyroid and Breast Surgery, \\ Affiliated Hospital of Zunyi Medical University, Zunyi, China \\ Contributions: (I) Conception and design: H Zhao, J Zhu; (II) Administrative support: C De Souza; (III) Provision of study materials or patients: \\ VE Kumar, R Nambiar, S Liu; (IV) Collection and assembly of data: D Hao, X Zhu, Y Luo; (V) Data analysis and interpretation: L Zhang; (VI) \\ Manuscript writing: All authors; (VII) Final approval of manuscript: All authors. \\ Correspondence to: Jingqiang Zhu. Department of Thyroid and Parathyroid Surgery, West China Hospital, Sichuan University, 37 Guoxue Alley, \\ Chengdu 610041, China. Email: zjq-wkys@163.com.
}

\begin{abstract}
Thyroid cancer (TC) is the most common endocrine malignancy, with high incidence rates in recent decades. Most TC cases have good prognoses, but a high risk of recurrence and metastases poses challenges, especially for patients with high-risk factors. Currently used prognostic markers for TC involve a combination of genetic factors and overexpressed proteins. Long non-coding RNAs (lncRNAs) regulate several integral biologic processes by playing key roles in the transcription of several downstream targets maintaining cellular behavior. Prior studies have revealed that lncRNAs promote tumor cell proliferation, invasion, metastasis, and angiogenesis, making them important targets for therapeutic intervention in cancer. While the exact molecular mechanisms underlying the role of lncRNAs in modulating TC progression and recurrence is still unclear, it is important to note that some lncRNAs are upregulated in certain cancers, while others are downregulated. In the present study, we review several key lncRNAs, their association with cancer progression, and the important roles they may play as tumor suppressors or tumor promoters in tumorigenesis. We discuss the potential mechanisms of lncRNA-mediated pathogenesis that can be targeted for the treatment of TC, the existing and potential benefits of using lncRNAs as diagnostic and prognostic measures for cancer detection, and tumor burden in patients.
\end{abstract}

Keywords: Thyroid cancer (TC); long non-coding RNAs (lncRNAs); tumor promoters

Submitted Nov 16, 2020. Accepted for publication Jan 26, 2021.

doi: 10.21037/atm-20-8191

View this article at: http://dx.doi.org/10.21037/atm-20-8191

\section{Introduction}

Thyroid cancer (TC) is the most common endocrine malignancy, with high incidence rates. According to the American Cancer Society's most recent statistics, it has been estimated that there will be 52,890 new cases of TC $(12,720$ in men and 40,170 in women) and about 2,180 deaths (1,040 men and 1,140 women) in the USA by the end of 2020. TC can be classified into 2 main subtypes based on gross histological features: differentiated and

\footnotetext{
$\wedge$ ORCID: 0000-0002-9087-0274.
} 
undifferentiated. Differentiated TC includes papillary thyroid carcinoma (PTC) and follicular thyroid carcinoma (FTC), which account for approximately $90 \%$ of all cases. Undifferentiated TC includes anaplastic thyroid carcinoma (ATC), which is the most aggressive form of TC and has poor clinical outcomes and limited treatment options. Medullary TC (MTC), which originates from parafollicular thyroid "C" cells are even less frequent, with incidence rates ranging from $3 \%$ to $5 \%$. Conventionally, surgical resection is the main therapeutic strategy, along with adjuvant therapies that include radioactive iodine therapy and hormone-based therapy. Most TC cases have good prognoses, but a high risk of recurrence and metastases poses challenges, especially for patients with high-risk factors. Prior research has revealed that the molecular pathogenesis of TC is governed by underlying dysregulated genetic and epigenetic determinants. It is imperative to identify biomarkers that can be accurately used to predict diagnosis, prognosis, and treatment response with high specificity and sensitivity in patients with TC. Long noncoding RNAs (lncRNAs) are one of the most important regulatory factors implicated in TC carcinogenesis (1). These evolutionarily distinct non-coding transcripts exist in lengths longer than 200 nucleotides (2). While some studies have shown that lncRNAs can play opposing roles and behave like oncogenes or tumor-suppressor genes during tumorigenesis, there is still no consensus about the biologic implications of these non-coding transcripts in the clinical setting (3). Prior studies have demonstrated the significant roles that lncRNAs play in fundamental cellular functions, such as pre-transcription, post-transcription, cell proliferation, invasion, differentiation, apoptosis, and migration (4). Increasing evidence suggests that lncRNAs also play a role in regulating cellular functionality and pathogenesis in TC (5). In addition, the expression of lncRNAs plays a role in modulating recurrence and survival rates in TC patients (6). For example, the lncRNA HOXA transcript at the distal tip (HOTTIP) knockdown inhibits cellular proliferation, invasion, and migration in vitro and in vivo (7). A recent study explored the high expression of metastasis-associated lung adenocarcinoma transcript 1 (MALAT1) and its correlation with tumor size, lymph node metastases, and World Health Organization (WHO) disease stage (8). These lncRNAs have received increasing attention as potential markers in the diagnosis and treatment evaluation of TC, especially for unclear cytological findings that cannot distinguish malignant from benign nodules. In the present study, we review some of the common lncRNAs and their association with prognoses in different cancer types. We further discuss their relevance and significance if adopted as a clinical measure in TC, and the role that these lncRNAs may play as targets for genetic or therapeutic intervention for disease prevention and control.

We present the following article in accordance with the Narrative Review reporting checklist (available at http:// dx.doi.org/10.21037/atm-20-8191).

\section{Existing prognostic markers for TC}

Currently used prognostic markers for TC involve a combination of genetic factors and overexpressed proteins. Well-differentiated thyroid carcinoma subtypes have good prognosis following the complete removal of the thyroid tumor, with a few cases of recurrence (9). Poorly differentiated thyroid carcinoma has a worst prognosis with ATC, with poor prognosis despite available treatment regimens (10). The overexpression of protein biomarkers has been studied, and galectin-3 (LGALS3) and FOXP3 have been identified as candidates for diagnosis and prognosis in differentiated thyroid carcinomas, with $L G A L S 3$ found to be associated with lymph node metastasis $(11,12)$. Genetic markers include mutations in oncogenes, such as $R A S$ and $B R A F$, and the promoter regions of telomerase reverse transcriptase (TERT). RET/PTC (rearrangement of RET gene) and $P A X 8 / P P A R \gamma$ chromosomal rearrangements have also been reported as markers for individuals exposed to radiation, with emphasis on the development of a more aggressive form of PTC $(13,14)$. Three variants of the mutation in RAS--KRAS, HRAS, and NRAS--have been reported in TC, with the NRAS codon 16 mutation found to be associated with distant metastasis in FTC (15). Mutations in the TERT promoter, which were found to be associated with malignancy in TC and in combination with BRAFV600E, were used to identify patients who could develop aggressive subtypes. BRAFV600E was found to be present in a majority of TC subtypes; however, it was not found to be associated with distant metastasis (16). Mutations in $B R A F$ were also reported to be a biomarker for prognosis associated with recurrence (17), while another study concluded $B R A F$ to be of minimal prognostic value in PTC (18). LGALS3 has been found to be unreliable as an exclusive prognostic marker (19-21). Conflicting studies on these biomarkers highlight the need for consistent and reliable prognostic indicators that can efficiently differentiate between TC subtypes and determine treatment regimens. 
Table 1 LncRNAs upregulated in TC

\begin{tabular}{|c|c|c|c|c|}
\hline LncRNAs & Tumor & Function & Molecular mechanisms & Source \\
\hline MALAT1 & MTC & Decreases cell proliferation and invasion & Regulates miR-21 & (24) \\
\hline SNHG15 & PTC & $\begin{array}{l}\text { Promotes cell growth and migration in papillary } \\
\text { thyroid carcinoma }\end{array}$ & $\begin{array}{l}\text { Regulates YAP1-Hippo signaling pathway } \\
\text { by sponging miR-200a-3p }\end{array}$ & $(25)$ \\
\hline SPRY4-IT & PTC & $\begin{array}{l}\text { Correlated with poor prognosis, and promotes } \\
\text { proliferative and migratory abilities of TC cells }\end{array}$ & Targets TGF- $\beta 1 /$ Smad signaling pathway & (26) \\
\hline Linc00210 & TC & $\begin{array}{l}\text { Augments proliferation, migration, and invasion of } \\
\text { TC cells }\end{array}$ & Modulates miR-195-5p/IGF1R/Akt axis & (28) \\
\hline UCA1 & TC & Promotes cell proliferation and the EMT phenotype & Via the UCA1/miR-15a axis & (29) \\
\hline CCAT1 & TC & Promotes cell proliferation, migration, and invasion & $\begin{array}{l}\text { Activate PI3K/Akt and MAPK signaling } \\
\text { pathways via downregulation of miR-143 }\end{array}$ & (30) \\
\hline XIST & TC & Modulates cell proliferation and tumor growth & $\begin{array}{l}\text { As a ceRNA for miR-34a through sponging } \\
\text { miR-34a, competing with MET for } \\
\text { miR-34a binding }\end{array}$ & (31) \\
\hline NEAT1 & $\mathrm{TC}$ & Accelerates thyroid cancer cell growth and metastasis & Regulates miRNA-214 expression & (32) \\
\hline
\end{tabular}

LncRNA, long non-coding RNA; TC, thyroid cancer; FTC, follicular thyroid carcinoma; PTC, papillary thyroid carcinoma; MTC, medullary TC.

\section{LncRNAs overexpressed in TC}

While the exact molecular mechanisms underlying the role of lncRNAs in modulating TC progression and recurrence is still unclear, it is important to note that some lncRNAs are upregulated in certain cancers, while others are downregulated. To understand the distinct roles each of these lncRNAs play in TC progression, we discuss the $\operatorname{lncRNAs}$ that are upregulated and those that are downregulated in different cancer types (Tables 1 and 2), along with the molecular mechanisms governing their regulatory behaviors. This information will help identify key patterns and phenotypic behaviors associated with each of these lncRNAs, therefore providing specific strategies for therapeutic intervention. A schematic describing the major mechanisms through which lncRNAs can regulate cell growth, metastasis, and cancer progression is shown in Figure 1.

\section{LncRNAs upregulated in TC}

\section{Colon cancer-associated transcript-1 (CCAT1)}

CCAT1 is a 2628-bp lncRNA that was initially identified in colon cancer. It is located at chromosome $8 \mathrm{q} 24.21$, and is in the vicinity of the proto-oncogene $c-M Y C$ (51). CCTA1 has 
Table 2 LncRNAs downregulated in TC

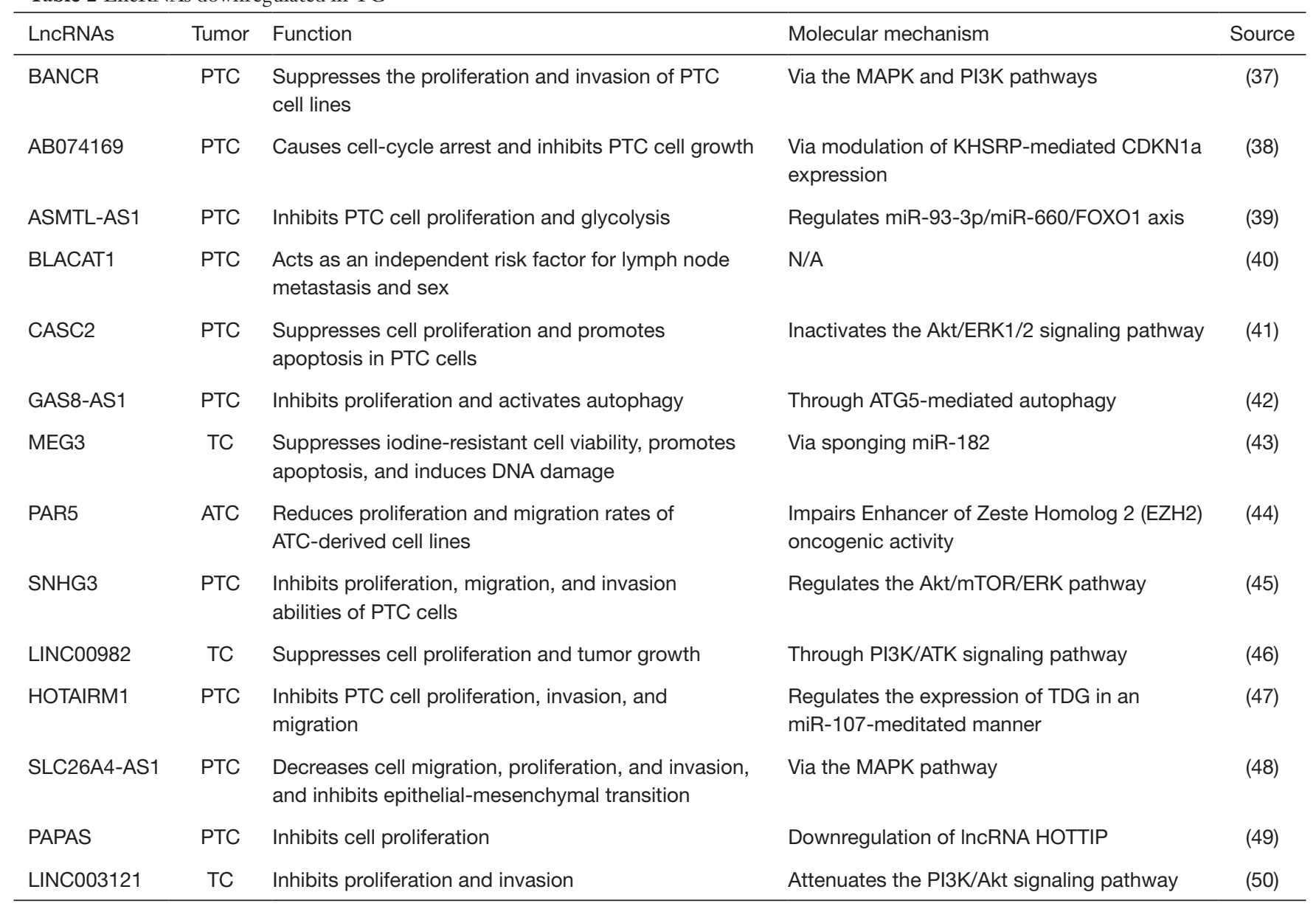

LncRNA, long non-coding RNA; TC, thyroid cancer; PTC, papillary thyroid carcinoma; ATC, anaplastic thyroid carcinoma.

been previously demonstrated to be significantly associated with tumor progression of different cancers, including PTC. Prior studies have reported CCAT1 to be highly expressed in the human TC cell line FTC-133, and CCAT1 suppression was found to reduce cell viability, proliferation, migration, invasion, and miR-143 expression (30). In addition, CCAT1 elevates apoptosis and vascular endothelial growth factor expression. Further studies have revealed that CCAT1 acts as a competitive endogenous RNA (ceRNA) to activate the phosphoinositide-3 kinase (PI3K)/Akt and mitogen-activated protein kinase (MAPK) signaling pathways via the inhibition of miR-143. In another recent study, CCAT1 was demonstrated to be one of the most significantly downregulated transcripts in ASH1L knockout cells. Further studies have shown that CCAT1 knockdown could suppress cell growth in ATC. ChIP-sequencing data analysis found that CCTA1 is likely involved in the regulation of ASH1L histone methyltransferase (52). These prior experimental data strongly suggest that CCTA1 exhibits oncogenic behavior in TC.

\section{H19}

H19, a 2.7-kb lncRNA, has been found to play a pivotal role in both embryonic development and tumorigenesis, and is located on chromosome 11p15.5 (53). H19 has been previously shown to be upregulated in tumor samples and in TC cell lines (22). The overexpression of $\mathrm{H} 19$ was observed to correlate significantly with proliferation, migration, and invasion, whereas the low expression of $\mathrm{H} 19$ reduced cell viability and invasion, inducing growth arrest in vitro and in vivo. Further studies found that $\mathrm{H} 19$ acts as ceRNA through competitively binding to miR-17-5p to upregulate its target YES1 to promote cell cycle progression in a TC 


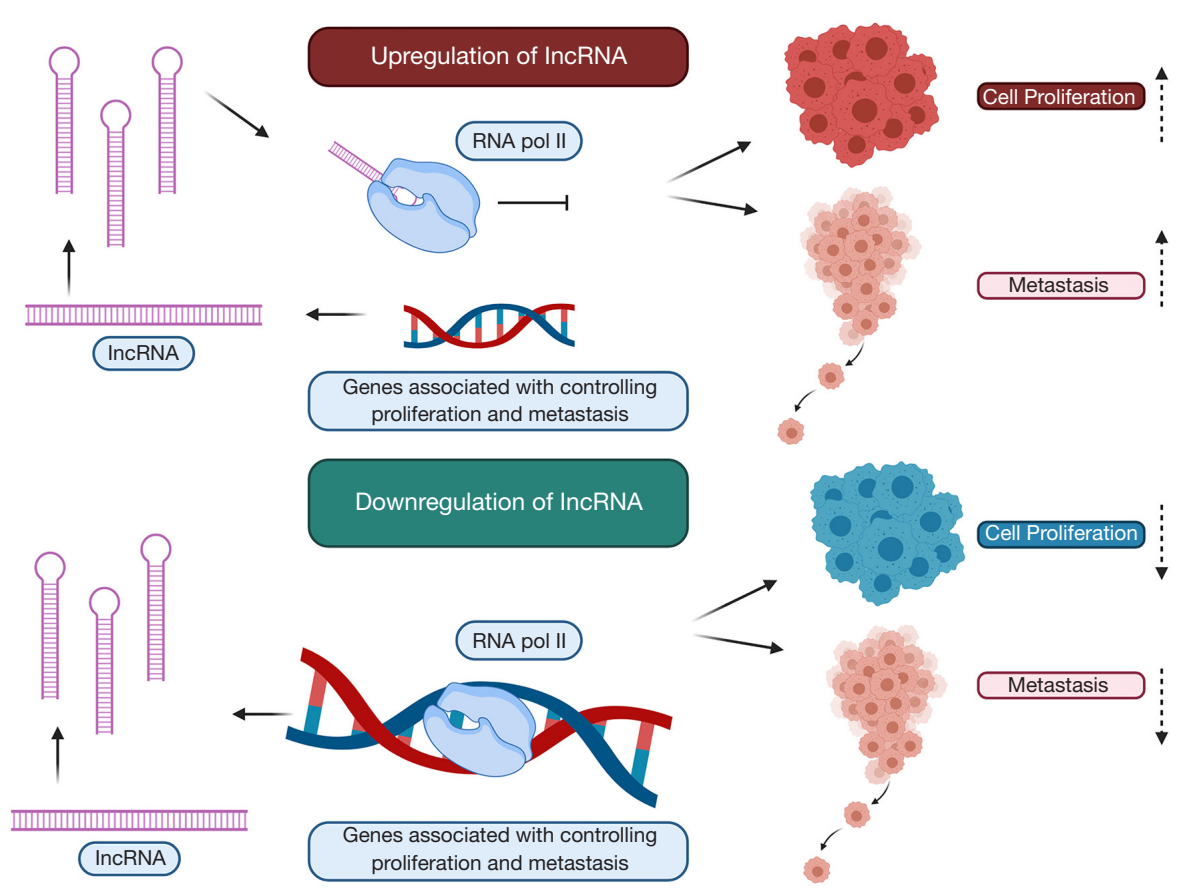

Figure 1 Long non-coding RNA (lncRNA) can transcriptionally regulate several key transcription factors and cell growth and invasion to promote cancer.

model. A recent study showed that the overexpression of H19 contributed to higher tumor burden in PTC (54). In addition, H19 promotes the epithelial-mesenchymal transition (EMT), mediating the migration and invasion of PTC cells. Other studies have found that H19 expression is upregulated in TC (55). Moreover, silencing H19 leads to increased levels of phosphorylated PI3 K and Akt, resulting in an inhibition of cell viability and higher levels of apoptosis. These studies indicate that H19 is another oncogenic regulator of TC.

\section{HLA complex P5 (HCP5)}

HCP5, an oncogenic lncRNA, has an important role in various types of cancers, including colon cancer $(56,57)$, cervical cancer $(58,59)$, and TC (60). HCP5 upregulation has been identified using next-generation sequencing technology in FTC (23). Moreover, the increased expression of HCP5 has been found to significantly promote cell proliferation, migration, invasiveness, and angiogenesis. Further studies have indicated that HCP5 acts as a ceRNA by competitively binding to miR-22-3p, miR-186-5p, and miR-216a-5p, promoting higher levels of ST6GAL2, resulting in increased cell proliferation and invasion. Similarly, Chen et al. reported that the expression of HCP5 is upregulated in ATC (60). The decreased expression of HCP5 inhibits cell viability and promotes higher apoptotic rates and caspase-3/7 activity. Further studies have showed that HCP5 binds to miR-128-3p and regulates the expression of miR-128-3p using luciferase reporter and RNA immunoprecipitation assays. These studies demonstrate that HCP5 acts as an oncogene in tumorigenesis, and stimulates invasion and metastases in tumor progression.

\section{HOTTIP}

HOTTIP is a 3764-nucleotide lncRNA that is encoded from a genomic region in the 5' tip of the HOXA locus. Prior studies have shown that the expression of HOTTIP is upregulated in PTC tissues, as well as cell lines (7). HOTTIP knockdown has been shown to suppress cell proliferation, invasion, and migration in vitro and in vivo. HOTTIP knockdown inhibits Akt1 expression and regulates miR-637 to inhibit cell proliferation, invasion, and migration in PTC cells. HOTTIP may be a worthy therapeutic target for malignancies. Other recent studies have shown that HOTTIP plays an oncogenic role in PTC 
via the negative regulation of miR-744-5p. This ultimately leads to elevated apoptosis. These studies suggest that HOTTIP is also a typical oncogenic lncRNA and may be an important therapeutic target in PTC (61).

\section{HOXA cluster antisense RNA2 (HOXA-AS2)}

HOXA-AS2 is a novel cancer-related lncRNA that has been demonstrated to be aberrantly expressed in various cancer types (62-64). In TC, the expression of HOXAAS2 is upregulated in PTC tissues (65). PTC cell growth was found to be inhibited through HOXA-AS2 knockdown in vitro and in vivo. PTC cell migration and invasion are promoted by HOXA-AS2 via the EMT phenotype. In addition, luciferase reporter assays have found that HOXA-AS2 could compete with miR-520c-3p at the 3 '-untranslated region (UTR) with a complementary binding site and inhibit the expression of miR-520c-3p. S100 calcium-binding protein A4 (S100A4) was confirmed as a downstream target of the miR-520c-3p by luciferase reporter assays. The data suggest that the HOXA-AS2/miR$520 \mathrm{c}-3 \mathrm{p} / \mathrm{S} 100 \mathrm{~A} 4$ axis may play a vital role in the regulation of PTC progression. A similar pattern was also observed in PTC cell lines. HOXA-AS2 overexpression was correlated with poor overall survival in patients with TC (66). This study had shown that HOXA-AS2 knockdown inhibits cellular proliferation, migration, and invasion, and accelerated apoptosis in PTC. The overexpression of HOXA-AS2 exhibited pro-oncogenic behavior. Moreover, the study found that HOXA-AS2 binds to miR-15a-5p and could upregulate HOXA3 expression. These studies demonstrate that HOXA-AS2 plays an important role in the progression of PTC, and could be a regulatory factor determining HOXA3 expression. Therefore, HOXA3 could also be a potential therapeutic target and biomarker for PTC diagnosis and prognosis.

\section{MALAT1}

MALAT1 is an onco-lncRNA that has been found to be overexpressed in several cancers (67-69). A previously published study established that MALAT1 was upregulated specifically in PTC tissues relative to normal tissues. A high expression of MALAT1 was found to correlate with tumor size, lymph node metastases, and WHO disease stage (8). Chu et al. analyzed the expression of MALAT1 in MTC and found that a high expression of MALAT1 in 37 (95\%) primary MTC and a strong expression of miR-21 in 17 (44\%) primary MTC in situ hybridization (70). Upregulated miR-21 and MALAT1 were found in MTC-derived cell lines compared with normal tissues. It was also shown that miR-21 and MALAT1 knockdown significantly increased cell proliferation and invasion. The data suggest that miR-21 and MALAT1 may regulate MTC progression. In a recent study, MALAT1 gene expression was significantly upregulated after dual MEK/Aurora kinase inhibitor "BI-847325" treatment in C643 and SW1736 cell lines. Further studies showed that Mcl1 and cyclin D1 expression were significantly reduced following BI-847325 treatment. Moreover, MALAT1 downregulated Mcl1 by competitively binding to and inhibiting miR-363-3p. These investigations show that MALAT1 plays a vital oncogenic function in TC (71).

\section{SNHG15}

LncRNA SNHG15 has been identified as a tumor facilitator in several types of cancers, including TC $(25,27,72-74)$. Wu et al. (25) reported that SNHG15 expression is significantly upregulated in PTC tissues and cell lines. In addition, SNHG15 expression was shown to be associated with the overall survival of PTC patients, and SNHG15 silencing led to the inhibition of cell growth and migration in PTC models. These studies indicate that SNHG15 upregulation can lead to the inactivation of the Hippo signaling pathway. Therefore, SNHG15 also acts as an oncogene in tumorigenesis and stimulates invasion and metastases in tumor progression.

\section{SPRY4-IT}

SPRY4-IT is an IncRNA that has been demonstrated to be a vital role in tumorigenesis associated with various cancer types (26,75). In a study by Zhou et al. (26), SPRY4-IT expression was found to be upregulated in TC tissues and cells. This elevated expression was found to be associated with poor prognosis. Studies have revealed that SPRY4IT silencing could suppress TC cell proliferation and migration, and other studies have shown that silenced SPRY4-IT promotes a higher expression of transforming growth factor- $\beta 1$ (TGF- $\beta 1$ ) and p-SMAD2/3, which are known regulators of cell invasion and migration. Conversely, TGF- $\beta 1$ could restore growth inhibition that had been induced by silencing SPRY4-IT1. These data suggest that SPRY4-IT contributes to the progression of tumor cell growth and acts via the TGF- $\beta 1 / \mathrm{SMAD}$ signaling pathway. 


\section{Urothelial carcinoma associated 1 (UCA1)}

UCA1, a 1.4-kb long transcription lncRNA, was first found in bladder cancer and is highly expressed in various carcinomas $(76,77)$. UCA1 expression is upregulated in PTC tissues and cells, and has been observed to be positively correlated with tumor size, tumor stage, and metastasis of PTC (31). UCA1 overexpression promotes cell proliferation and invasion, and suppresses apoptosis in TC cells via the Wnt pathway. UCA1 acts as an oncogene in tumorigenesis, and stimulates invasion in tumor progression. Many studies have revealed that UCA1 elevates tumorigenesis mainly through binding to miRNAs, activating several vital signaling pathways and altering epigenetic and transcriptional regulation $(78,79)$. In TC, the expression of UCA1 is significantly upregulated in PTC tissue compared with normal tissue (80). Through loss-of-function analysis, UCA1 knockdown was found to significantly inhibit PTC cell viability and promote the expression of BRD4. UCA1 was also found to promote the progression of PTC through sponging miRNA-204 and enhancing BRD4 expression. Li et al. found that the UCA1/ miR-15a axis is involved in mediating EMT in PTC (29). In ATC, UCA1 promotes cancer progression through miR-135a-mediated $c-M Y C$ activation (81). Another study revealed that UCA1 knockdown inhibits cell proliferation and invasion by modulating the miR-204/IGFBP5 axis in PTC (82). These studies demonstrate that UCA1 plays a vital role in the progression of TC, and may be a potential target for TC treatment.

\section{Mechanisms underlying IncRNA-mediated metastasis in PTC tumors}

EMT is implicated in the progression of FTC and PTC converting to PDTC and ATC by conferring stem-like properties to cancer stem cells (83). To enter the blood circulation, during EMT epithelial cancer cells undergo a decrease in cell-cell adhesion and cell polarity and an increase in mesenchymal features, and migrate to secondary areas; upon arrival in the secondary site, they invade the tissue through the MET, which is the reverse process of EMT (84). In cancer cells, changes in gene expression accompanying the EMT-MET process is controlled by multiple transcription factors, such as SNAIL, SLUG, and ZEB1 (85); growth factors and their associated signaling proteins (86); epigenetic regulators; and the tumor microenvironment. LncRNAs interacting with target genes involved in the EMT-MET process have also been recently reported in the literature (87). LncRNAs regulate gene expression by functioning as signaling molecules inducing gene expression, decoys that sequester transcription factors, guides that recruit RNA binding proteins, and scaffolds that form protein complexes (88). In PTC, lncRNAs have been reported to regulate gene expression networks by a variety of methods, such as directly binding to miRNAs and preventing their interaction with target genes or proteins, directly binding to target genes, post-transcriptional epigenetic modification by recruiting histone modifiers [e.g., enhancer of zeste homolog 2 (EZH2)], and regulating proteins involved in signaling pathways (e.g., TGF- $\beta 1$, PI3K/Akt, Wnt/ $\beta$-catenin) (89). Given this range of mechanisms of lncRNAs in PTC, identification and further characterization of these molecules can pave the way for potential diagnostic, prognostic, and therapeutic targets.

\section{LncRNAs downregulated in TC}

Understanding the molecular underpinnings of how these regulatory lncRNAs are downregulated in TC is of importance to develop novel diagnostic and prognostic markers that can serve to alter tumor functionality.

\section{BRAF-activated non-coding RNA (BANCR)}

BANCR is a 693-bp lncRNA that was first identified by Flockhart in 2012 (90). BANCR expression is detected in many tumors, including melanoma (91,92), gastric cancer (93), lung cancer (94), and endometrial cancer (95). Liao et al. (96) analyzed BANCR expression in 92 patients with PTC and normal thyroid epithelial tissues using quantitative reverse transcription polymerase chain reaction. BANCR was found to be downregulated in PTC tissue compared with control tissue. Decreased BANCR expression is associated with tumor size, multifocal lesions, and tumor stages. Furthermore, increased BANCR expression was also found to decrease PTC cell proliferation and increase apoptosis. BANCR expression also inactivates ERK1/2 and P38 inhibiting tumorigenesis in PTC. A previous study reported BANCR to be significantly downregulated in PTC tissues compared with matched normal thyroid tissues, and it was found to be strongly correlated with lymph node metastasis. Functional studies have indicated that the overexpression of BANCR leads to G2/M cell-cycle arrest and increased apoptosis (37). In addition, the MAPK and PI3K/Akt pathways are aberrantly activated by western blotting, 


\section{Page 8 of 16}

resulting in decreased cancer cell proliferation and invasion. These studies demonstrate that BANCR could play an integral role as a tumor suppressor in TC.

\section{AB074169}

AB074169 is a novel lncRNA. AB074169 expression has been previously shown to be significantly downregulated in PTC (38). The decreased expression of AB074169 was found to be related to $\mathrm{CpG}$ hypermethylation within its gene promoter. Through loss- and gain-of-function analyses, AB074169 overexpression was found to cause cellcycle arrest and inhibit the growth of PTC cells. However, AB074169 knockdown promoted cell proliferation. Further studies demonstrated that AB074169 binds to the splicing regulatory protein (KHSRP) and inhibits the expression of KHSRP, therefore increasing CDKN1a (p21) expression and decreasing CDK2 expression to inhibit cell proliferation. These data suggest that $\mathrm{AB} 074169$ acts as a tumor suppressor during PTC tumorigenesis.

\section{ASMTL antisense RNA1 (ASMTL-AS1)}

ASMTL-AS1 is a novel lncRNA that is significantly downregulated in PTC. A low expression of ASMTLAS1 has been found to be positively associated with larger tumor size, advanced clinical stages, and an unfavorable outcome (39). Gain-of-function assays have demonstrated that elevated ASMTL-AS1 promotes TPC cell proliferation and glycolysis. Loss-of-function analysis have found that ASMTL-AS1 knockdown has the opposite effect. Luciferase reporter gene demonstrated that ASMTL-AS1 also promotes the expression of FOXO1 via sponging miR-93-3p and miR-660, and inhibits glycolysis and tumorigenesis. In addition, FOXO1 is capable of binding to the ASMTLAS1 promoter to elevate ASMTL-AS1 expression, which leads to a feedback regulation loop. The regulatory axis of ASMTL-AS1/miR-93-3p/miR-660/FOXO1 was identified in the animal study. This study concluded that ASMTLAS1 is likely to play a role as a tumor suppressor gene and could be used as a potential predictor in PTC patients.

\section{Bladder cancer-associated transcript 1 (BLACAT1)}

The BLACAT1 lncRNA was first found in bladder cancer and is also expressed in several other of human cancers. In

\section{Zhao et al. LncRNAs as predictors of prognosis in thyroid cancer}

PTC, BLACAT1 expression was found to be significantly downregulated in the plasma of 87 PTC patients (case group) compared with 36 patients with nodular goiter (control group) (40). Low plasma BLACAT1 expression is correlated with lymph node metastasis. A multivariate analysis demonstrated that BLACAT1 is an independent risk factor for lymph node metastasis and sex. Collectively, these data indicate that BLACAT1 acts as a vital tumor suppressor gene, and a potential biomarker for the prediction of prognosis in PTC.

\section{Cancer susceptibility candidate 2 (CASC2)}

CASC2 is a lncRNA that been identified as a tumor suppressor gene in several types of cancers, including colorectal, lung, and renal (97-99). A recent study showed that expression was significantly downregulated in PTC tissues compared with adjacent normal tissues (41). Gainof-function assays have demonstrated that elevated CASC2 expression inhibits cell proliferation and increases apoptosis in PTC cells. CASC2 overexpression leads to the inactivation of protein kinase $\mathrm{B} / \mathrm{Akt}$ and extracellular signalregulated kinase $1 / 2$. Other studies have found that MAPK (MEK) inhibitor U0126 or AktT1/2/3 inhibitor MK-2206 $2 \mathrm{HCl}$ enhances the regulatory effects of CASC2 on the biologic behavior of PTC. Similarly, CASC2overexpression inhibits tumor growth in PTC cells in vivo. These observations suggest that CASC2 significantly inhibits tumorigenesis in PTC. Therefore, CASC2 shows promise as a potential prognostic marker and therapeutic target.

\section{HOX antisense intergenic RNA myeloid 1 (HOTAIRM1)}

HOTAIRM is a lncRNA that is associated with various cancers (100-102). HOTAIRM1 expression is significantly downregulated in PTC. tissues and the low expression of HOTAIRM1 is associated with lymph node metastasis and advanced TNM stage. Moreover, gain-of-function assays have demonstrated that elevated HOTAIRM1 inhibits PTC cell proliferation, invasion, and migration in vitro. Subsequent studies have also confirmed that HOTAIRM1 competes with endogenous miR-107. In addition, these studies also demonstrated that HOTAIRM1 regulates the expression of TDG in a miR-107-meditated manner (47). These data indicate that HOTAIRM1 acts as a tumor suppressor gene in PTC, and may serve as a therapeutic 
target for PTC patients.

\section{Growth arrest-specific 5 (GAS5)}

GAS5 is a non-coding gene that hosts several small nucleolar RNAs. It was originally isolated from mouse NIH 3T3 cells using subtraction hybridization. GAS5 is induced by cellular stressors, such as serum starvation and cell-cell contact inhibition (103). Guo et al. analyzed the expression of GAS5 in 212 TC patients and 61 benign thyroid tumor patients (104). The patients were divided into high-risk and low-risk groups according to the MACIS, AGES, and AMES prognostic scoring systems. It was found that the expression of GAS5 was downregulated in TC tissues compared with benign tissues. This GAS5 downregulation was significantly associated with TNM staging, lymph node metastasis, and multiple cancer foci of TC. GAS5 expression decreased in the MACIS high-risk cohort compared with the AMES low-risk patients. Similarly, TC patients with high GAS5 expression had a better disease outcome compared with TC patients with low GAS expression. These data suggest that GAS5 can function as a biomarker for the diagnosis and prognosis of TC.

\section{LncRNA Prader Willi/Angelman region RNA5 (PAR5)}

PAR5, also known as PWAR5, plays an important role in various types of cancers, including glioma and ATC $(44,105)$. Pellecchia et al. (105) investigated the lncRNA expression profiles of 9 ATC samples compared with 5 normal thyroid tissues and identified 19 upregulated and 28 downregulated lncRNAs with a fold change $>1.1$ or $<-1.1$. Other studies found that the expression of PAR5 was significantly downregulated in ATC samples, and that the downregulation of PAR5 contributes to the restoration of reduced proliferation and migration (105). Studies also have found that PAR5 impacts EZH2 oncogenic activity by impacting its transactivation potential on E-cadherin. These results suggest that PAR5 has typical tumor suppressive behavior in TC.

\section{SNHG3}

SNHG3 is a novel lncRNA expressed in various types of cancers, including PTC $(45,106,107)$. The expression of SNHG3 has been reported to be significantly downregulated in PTC tissues and cell lines (45), and was observed to be significantly correlated with TNM stage and poor prognosis of PTC patients. SNHG3 depletion promotes proliferation, migration, and invasion in PTC cells. Similarly, silenced SNHG3 was found to promote malignant progression in tumor xenograft models. Further analyses demonstrated that SNHG3 knockout activates the Akt/mTOR/ERK pathway in PTC cell lines. These effects could then be rescued using the mTOR inhibitor AZD8055. These findings indicate that SNHG3 can act as a tumor suppressor gene in PTC, and might serve as a promising candidate for target therapy of PTC.

\section{GAS8-AS1}

GAS8-AS1 is the second most frequently altered gene, after the $B R A F$ gene in PTC. Previous studies have indicated that $G A S 8-A S 1$ functions as a tumor suppressor in PTC. In a recent study (42), GAS8-AS1 expression was found to be downregulated in PTC cells compared with a normal thyroid cell line. Gain-of-function studies have demonstrated that $G A S 8$-AS1 overexpression suppresses cell proliferation, increases the ratio of LC3-II/LC3-I, and reduces p62 expression, whereas GAS8-AS1 the knockdown is pro-oncogenic. GAS8-AS1 overexpression produces higher levels of LC3 staining and increases the expression of autophagosomes. Autophagy-related gene 5 was found to be significantly upregulated by $G A S 8-A S 1$ overexpression and downregulated by $G A S 8-A S 1$ knockdown. These data indicate that $G A S 8$ - $A S 1$ can modulate cell death in PTC via the autophagy pathway. Chen et al. found that the GAS8$A S 1$ was downregulated in PTC cell lines and suppressed the cell proliferation and cycle of PTC cells. Further studies showed that $G A S 8-A S 1$ regulates a downstream target of miR-135b-5p, cyclin G2, whereas overexpressed GAS8$A S 1$ inhibits tumor formation and suppresses PTC cell growth (108). These studies demonstrate that GAS8-AS1 inhibits PTC cell growth via the miR-135b-5p/CCND2 axis, and acts as a tumor suppressor gene in PTC.

\section{Maternally expressed gene 3 (MEG3)}

MEG3 is an imprinted gene belonging to the imprinted DLK1-MEG3 locus located at chromosome 14q32.3 in humans. Its mouse ortholog, MEG3, also known as gene trap locus 2 (Gt12), is located at distal chromosome 12 (109). A recent study showed that a low expression of MEG3 was found in TC tissues, and low MEG3 expression was positively correlated with the low cumulative survival rate in PTC patients under iodine treatment. Further studies 
found that MEG3 overexpression inhibits iodine-resistant cell viability, elevates the level of apoptosis, and induces DNA damage. In addition, MEG3 leads to the sponging of miR-182, and MEG3 knockdown substantially inhibits the anti-cancer functions of anti-miR-182. These experiments indicate that MEG3 could be a suitable target for TC patients with iodine resistance (43). MEG3 expression was found to be significantly downregulated in PTC tissues with lymph node metastasis compared with primary TC. Moreover, the low expression of MEG3 is associated with relative lymph node metastasis. MEG3 inhibits the migration and invasion of PTC cells. Further studies have demonstrated that MEG3 negatively regulates posttranscriptional processing through a specific target site within the 3 'UTR.

\section{LncRNAs as diagnostic and prognostic markers in TC}

We previously discussed the role of current genetic markers as prognostic and diagnostic markers for TC patients. It is also important to focus on lncRNAs as novel targets that show promise for their benefits as prognostic or diagnostic markers in TC progression and recurrence. Currently, the cytological evaluation of fine-needle aspiration (FNA) of thyroid nodules is the standard procedure to determine the need for surgical resection in TC patients. However, there are significant drawbacks in the use of FNA, and studies have reported the use of microarray analysis for FNA (110). Several lncRNAs have been described as significantly specific for TC. The differential expressions of those lncRNAs have also been found to be cells, tissue, and plasma in the TC.

As discussed earlier, some lncRNAs, such as CCAT1, H19, HCP5, HOTTIP, , MALAT1, HOXA-AS2, SNHG15, SPRY4-IT, and UCA1, have been found to be upregulated in TC $(7,23,25,26,30,42,70,111,112)$, whereas others, such as BANCR, AB074169, ASMTL-AS1, BLACAT1, CASC2, HOTAIRM1, GAS5, PAR5, SNHG3, GAS8-AS1, and MEG3 are downregulated (38-40,43$45,47,104,108,112,113)$. This unique biologic behavior exhibited by different groups of IncRNAs shows value for the prognostic and diagnostic determination in TC patients. However, further research is required to elucidate the mechanisms of action through which each of these lncRNAs exerts their effects. Further development, characterization, and standardization are essential if lncRNAs are to be used as prognostic markers in the clinical setting.
Most patients with TC are treated with surgical resection, thyroid hormone therapy, chemotherapy, and radiotherapy. However, recurrence due to metastasis can still occurs despite treatment. This recurrence is often linked to EMT, and therefore, identifying lncRNAs specific for EMT may help diagnose the disease earlier. As metastasis is reported to occur due to genetic changes during the development of primary tumors, identifying metastatic-specific biomarkers can assist in the early detection of metastatic cancers (114). LncRNAs H19, HOXA-AS2, and UCA1 have been reported to affect the invasive nature of TC cells. The clinical significance of some lncRNAs as prognostic determinants in clinical studies are described in Table 3.

\section{Discussion}

LncRNAs regulate various mechanisms involved in tumor initiation, progression, and metastasis (87). Due to spatiotemporal expression and plasma stability, the detection of circulating lncRNAs as prognostic and diagnostic biomarkers for TC is developing as a viable field of research. Moreover, lncRNAs detection in circulating fluids provides a non-invasive approach, avoiding the need for invasive biopsies (118). Despite these advantages, there are still challenges that affect the use of lncRNAs as biomarkers, including factors affecting the stability and bioavailability of lncRNAs in fluids and lncRNA extraction (89).

The targeting of lncRNAs through RNAi technology, anti-sense oligonucleotides (ASO) and small molecule inhibitors has also been investigated as a therapeutic approach in other cancers $(119,120)$. For instance, the subcutaneous injection of ASO targeting MALAT1 has been shown to block lung metastasis in mouse xenograft models. HOTAIR targeting small molecule inhibitor AC1NOD4Q has been shown to inhibit the binding of HOTAIR with EZH2, and reduces cancer metastasis in vitro and in orthotopic breast cancer models (120). At the same time, bioinformatics and database are used as important tools to predict co-genes and ce-RNA in the researches of lncRNA. In particular, there has been significant research demonstrating the presence of specific lncRNAs associated with various tumor mechanisms and disease stage (121). Currently, the mechanisms involved in lncRNA production, transportation, binding partners, and targets are not well understood, and further research is required to enable the clinical use of lncRNAs as biomarkers and potential therapeutic targets.

In the present study, we provided a comprehensive 
Table 3 Clinical significance of lncRNAs as prognostic markers in TC patients

\begin{tabular}{|c|c|c|c|c|}
\hline LncRNA & Tumor & Samples & Clinical significance & Source \\
\hline CASC2 & $\mathrm{TC}$ & $\begin{array}{l}172 \text { thyroid } \\
\text { carcinoma tissues }\end{array}$ & Correlates with multifocality and advanced TNM stage & $(41)$ \\
\hline LINC02454 & PTC & 104 PTC tissues & $\begin{array}{l}\text { Closely related to tumor size, } T \text { stage, lymph node metastasis and } \\
\text { disease-free survival. }\end{array}$ & $(115)$ \\
\hline $\mathrm{H} 19$ & PTC & 410 PTC patients & $\begin{array}{l}\text { Associated with patient age, tumor size, extrathyroidal extension, } \\
\text { pathological lateral node metastasis }(\mathrm{pN} 1 \mathrm{~b}) \text {, histological aggressive type, } \\
\text { and poorer disease-free survival }(\mathrm{P}<0.0001) \text {. }\end{array}$ & $(116)$ \\
\hline MIR22HG & ATC & $\begin{array}{l}9 \text { ATC patients and } \\
20 \text { PTC }\end{array}$ & $\begin{array}{l}\text { Significantly related to higher age, lymph node metastasis status, residual } \\
\text { tumor status, } \mathrm{N} \text { stage, grade, and T stage in TC }\end{array}$ & $(91)$ \\
\hline SPRY4-IT & $\mathrm{TC}$ & $80 \mathrm{TC}$ tissues & Correlated with poor prognosis & $(26)$ \\
\hline
\end{tabular}

LncRNA, long non-coding RNA; TC, thyroid cancer; PTC, papillary thyroid carcinoma; ATC, anaplastic thyroid carcinoma.

description of the lncRNAs involved in TC and their respective mechanisms of action during cancer growth and development. We also discussed their use as prognostic and diagnostic markers and the challenges in enabling lncRNAs to be used as prognostic markers in the clinical setting, as well as strategies to overcome these challenges. Further studies are warranted to better understand the role of lncRNAs in EMT, prevent the recurrence of TC and design effective treatment strategies.

\section{Acknowledgments}

The authors acknowledge BioRender.com for providing Figure 1.

Funding: The present study was funded by West China Hospital, Sichuan University (No. ZY2017309).

\section{Footnote}

Reporting Checklist: The authors have completed the Narrative Review reporting checklist. Available at http:// dx.doi.org/10.21037/atm-20-8191

Conflicts of Interest: All authors have completed the ICMJE uniform disclosure form (available at http://dx.doi. org/10.21037/atm-20-8191). The authors have no conflicts of interest to declare.

Ethical Statement: The authors are accountable for all aspects of the work in ensuring that questions related to the accuracy or integrity of any part of the work are appropriately investigated and resolved.

Open Access Statement: This is an Open Access article distributed in accordance with the Creative Commons Attribution-NonCommercial-NoDerivs 4.0 International License (CC BY-NC-ND 4.0), which permits the noncommercial replication and distribution of the article with the strict proviso that no changes or edits are made and the original work is properly cited (including links to both the formal publication through the relevant DOI and the license). See: https://creativecommons.org/licenses/by-nc-nd/4.0/.

\section{References}

1. Kentwell J, Gundara JS, Sidhu SB. Noncoding RNAs in endocrine malignancy. Oncologist 2014;19:483.

2. Kraczkowska W, Jagodziński PP. The Long Non-Coding RNA Landscape of Atherosclerotic Plaques. Mol Diagn Ther 2019;23:735-49.

3. Bush SJ, Muriuki C, McCulloch MEB, et al. Cross-species inference of long non-coding RNAs greatly expands the 
ruminant transcriptome. Genet Sel Evol 2018;50:20.

4. Guo C, Qi Y, Qu J, et al. Pathophysiological Functions of the lncRNA TUG1. Curr Pharm Des 2020;26:688-700.

5. Sarropoulos I, Marin R, Cardoso-Moreira M, et al. Developmental dynamics of lncRNAs across mammalian organs and species. Nature 2019;571:510-4.

6. Rao Y, Liu H, Yan X, et al. In Silico Analysis Identifies Differently Expressed lncRNAs as Novel Biomarkers for the Prognosis of Thyroid Cancer. Comput Math Methods Med. 2020;2020:3651051.

7. Yuan Q, Liu Y, Fan Y, et al. LncRNA HOTTIP promotes papillary thyroid carcinoma cell proliferation, invasion and migration by regulating miR-637. Int J Biochem Cell Biol 2018;98:1-9.

8. Liu J, Dong H, Yang Y, et al. Upregulation of long noncoding RNA MALAT1 in papillary thyroid cancer and its diagnostic value. Future Oncol 2018;14:3015-22.

9. Gillanders SL, O'Neill JP. Prognostic markers in well differentiated papillary and follicular thyroid cancer (WDTC). Eur J Surg Oncol 2018;44:286-96.

10. Lim SM, Shin SJ, Chung WY, et al. Treatment outcome of patients with anaplastic thyroid cancer: a single center experience. Yonsei Med J 2012;53:352-7.

11. Tang W, Huang C, Tang C, et al. Galectin-3 may serve as a potential marker for diagnosis and prognosis in papillary thyroid carcinoma: a meta-analysis. Onco Targets Ther 2016;9:455-60.

12. Cunha LL, Morari EC, Nonogaki S, et al. Foxp3 expression is associated with aggressiveness in differentiated thyroid carcinomas. Clinics (Sao Paulo) 2012;67:483-8.

13. Romei C, Elisei R. RET/PTC translocations and clinico-pathological features in human papillary thyroid carcinoma. Front Endocrinol (Lausanne) 2012;3:54.

14. Raman P, Koenig RJ. Pax-8-PPAR- $\gamma$ fusion protein in thyroid carcinoma. Nat Rev Endocrinol 2014;10:616-23.

15. Howell GM, Hodak SP, Yip L. RAS mutations in thyroid cancer. Oncologist 2013;18:926.

16. Su X, Jiang X, Wang W, et al. Association of telomerase reverse transcriptase promoter mutations with clinicopathological features and prognosis of thyroid cancer: a meta-analysis. Onco Targets Ther 2016;9:6965-76.

17. Xing M, Westra WH, Tufano RP, et al. BRAF mutation predicts a poorer clinical prognosis for papillary thyroid cancer. J Clin Endocrinol Metab 2005;90:6373-9.

18. Henke LE, Pfeifer JD, Ma C, et al. BRAF mutation is not predictive of long-term outcome in papillary thyroid carcinoma. Cancer Med 2015;4:791-9.
19. Dong R, Zhang M, Hu Q, et al. Galectin-3 as a novel biomarker for disease diagnosis and a target for therapy (Review). Int J Mol Med 2018;41:599-614.

20. Liu Z, Li X, Shi L, et al. Cytokeratin 19, thyroperoxidase, HBME-1 and galectin-3 in evaluation of aggressive behavior of papillary thyroid carcinoma. Int J Clin Exp Med 2014;7:2304-8.

21. Lee YM, Lee JB. Prognostic value of epidermal growth factor receptor, p53 and galectin-3 expression in papillary thyroid carcinoma. J Int Med Res 2013;41:825-34.

22. Liu L, Yang J, Zhu X, et al. Long noncoding RNA H19 competitively binds miR-17-5p to regulate YES 1 expression in thyroid cancer. FEBS J 2016;283:2326-39.

23. Liang L, Xu J, Wang M, et al. LncRNA HCP5 promotes follicular thyroid carcinoma progression via miRNAs sponge. Cell Death Dis 2018;9:372.

24. Wang KC, Yang YW, Liu B, et al. A long noncoding RNA maintains active chromatin to coordinate homeotic gene expression. Nature 2011;472:120-4.

25. Wu DM, Wang S, Wen X, et al. LncRNA SNHG15 acts as a ceRNA to regulate YAP1-Hippo signaling pathway by sponging miR-200a-3p in papillary thyroid carcinoma. Cell Death Dis 2018;9:947.

26. Zhou H, Sun Z, Li S, et al. LncRNA SPRY4-IT was concerned with the poor prognosis and contributed to the progression of thyroid cancer. Cancer Gene Ther 2018;25:39-46.

27. Huang J, Pan B, Xia G, et al. LncRNA SNHG15 regulates EGFR-TKI acquired resistance in lung adenocarcinoma through sponging miR-451 to upregulate MDR-1. Cell Death Dis 2020;11:525.

28. Du P, Liu F, Liu Y, et al. Linc00210 enhances the malignancy of thyroid cancer cells by modulating miR-195-5p/IGF1R/Akt axis. J Cell Physiol 2020;235:1001-12.

29. Li D, Hao S, Zhang J. Long non-coding RNA UCA1 exerts growth modulation by miR-15a in human thyroid cancer TPC-1 cells. Artif Cells Nanomed Biotechnol 2019;47:1815-22.

30. Yang T, Zhai H, Yan R, et al. lncRNA CCAT1 promotes cell proliferation, migration, and invasion by downregulation of miR-143 in FTC-133 thyroid carcinoma cell line. Braz J Med Biol Res 2018;51:e7046.

31. Lu HW, Liu XD. UCA1 promotes papillary thyroid carcinoma development by stimulating cell proliferation via Wnt pathway. Eur Rev Med Pharmacol Sci 2018;22:5576-82.

32. Li JH, Zhang SQ, Qiu XG, et al. Long non-coding RNA 
NEAT1 promotes malignant progression of thyroid carcinoma by regulating miRNA-214. Int J Oncol 2017;50:708-16.

33. Xiang J, Guan Y, Bhandari A, et al. LINC00511 influences cellular proliferation through cyclin-dependent kinases in papillary thyroid carcinoma. J Cancer 2020;11:450-9.

34. Yang C, Liu Z, Chang X, et al. NR2F1-AS1 regulated miR-423-5p/SOX12 to promote proliferation and invasion of papillary thyroid carcinoma. J Cell Biochem 2020;121:2009-18.

35. Sun Z, Guo X, Zang M, et al. Long non-coding RNA LINC00152 promotes cell growth and invasion of papillary thyroid carcinoma by regulating the miR-497/ BDNF axis. J Cell Physiol 2019;234:1336-45.

36. Liang M, Jia J, Chen L, et al. LncRNA MCM3AP-AS1 promotes proliferation and invasion through regulating miR-211-5p/SPARC axis in papillary thyroid cancer. Endocrine 2019;65:318-26.

37. Zhang J, Du Y, Zhang X, et al. Downregulation of BANCR promotes aggressiveness in papillary thyroid cancer via the MAPK and PI3K pathways. J Cancer 2018;9:1318.

38. Gou Q, Gao L, Nie X, et al. Long noncoding RNA AB074169 inhibits cell proliferation via modulation of KHSRP-mediated CDKN1a expression in papillary thyroid carcinoma. Cancer Res 2018;78:4163-74.

39. Feng Z, Chen R, Huang N, et al. Long non-coding RNA ASMTL-AS1 inhibits tumor growth and glycolysis by regulating the miR-93-3p/miR-660/FOXO1 axis in papillary thyroid carcinoma. Life Sci 2020;244:117298.

40. Liao D, Lv G, Wang T, et al. Prognostic value of long non-coding RNA BLACAT1 in patients with papillary thyroid carcinoma. Cancer Cell Int 2018;18:47.

41. Xiong X, Zhu H, Chen X. Low expression of long noncoding RNA CASC2 indicates a poor prognosis and promotes tumorigenesis in thyroid carcinoma. Biomed Pharmacother 2017;93:391-7.

42. Qin $Y$, Sun W, Zhang H, et al. LncRNA GAS8-AS1 inhibits cell proliferation through ATG5-mediated autophagy in papillary thyroid cancer. Endocrine 2018;59:555-64.

43. Liu Y, Yue P, Zhou T, et al. LncRNA MEG3 enhances 131I sensitivity in thyroid carcinoma via sponging miR182. Biomed Pharmacother 2018;105:1232-9.

44. Wang XP, Shan C, Deng XL, et al. Long non-coding RNA PAR5 inhibits the proliferation and progression of glioma through interaction with EZH2. Oncol Rep 2017;38:3177-86.
45. Duan $Y$, Wang Z, Xu L, et al. lncRNA SNHG3 acts as a novel Tumor Suppressor and regulates Tumor Proliferation and Metastasis via AKT/mTOR/ERK pathway in Papillary Thyroid Carcinoma. J Cancer 2020;11:3492.

46. Xu D, Yu J, Zhuang S, et al. Overexpression of long noncoding RNA LINC00982 suppresses cell proliferation and tumor growth of papillary thyroid carcinoma through PI3K-ATK signaling pathway. Biosci Rep 2019;39:BSR20191210

47. Li D, Chai L, Yu X, et al. The HOTAIRM1/miR107/TDG axis regulates papillary thyroid cancer cell proliferation and invasion. Cell Death Dis 2020;11:227.

48. Wang DP, Tang XZ, Liang QK, et al. Overexpression of long noncoding RNA SLC26A4-AS1 inhibits the epithelial-mesenchymal transition via the MAPK pathway in papillary thyroid carcinoma. J Cell Physiol 2020;235:2403-13.

49. Xiao J, Bing Z, Xiao G, et al. Long non-coding (lnc) RNA PAPAS overexpression inhibits tumor growth in papillary thyroid carcinoma by downregulating lncRNA HOTTIP. Oncol Lett 2020;19:2281-5.

50. Min X, Liu K, Zhu H, et al. Long Noncoding RNA LINC003121 Inhibits Proliferation and Invasion of Thyroid Cancer Cells by Suppression of the Phosphatidylinositol-3-Kinase (PI3K)/Akt Signaling Pathway. Med Sci Monit 2018;24:4592-601.

51. Xin Y, Li Z, Shen J, et al. CCAT 1: a pivotal oncogenic long non-coding RNA in human cancers. Cell Prolif 2016;49:255-60.

52. Xu B, Qin T, Yu J, et al. Novel role of ASH1L histone methyltransferase in anaplastic thyroid carcinoma. J Biol Chem 2020;295:8834-45.

53. Raveh E, Matouk IJ, Gilon M, et al. The H19 Long non-coding RNA in cancer initiation, progression and metastasis-a proposed unifying theory. Mol Cancer 2015;14:184.

54. Liang WQ, Zeng D, Chen CF, et al. Long noncoding RNA H19 is a critical oncogenic driver and contributes to epithelial-mesenchymal transition in papillary thyroid carcinoma. Cancer Manag Res 2019;11:2059-72.

55. Li X, Li Q, Jin X, et al. Long non-coding RNA H19 knockdown inhibits the cell viability and promotes apoptosis of thyroid cancer cells through regulating the PI3K/AKT pathway. Exp Ther Med 2019;18:1863-9.

56. Yun WK, Hu YM, Zhao CB, et al. HCP5 promotes colon cancer development by activating AP1G1 via PI3K/AKT pathway. Eur Rev Med Pharmacol Sci 2019;23:2786-93.

57. Bai N, Ma Y, Zhao J, et al. Knockdown of lncRNA 
HCP5 Suppresses the Progression of Colorectal Cancer by miR-299-3p/PFN1/AKT Axis. Cancer Manag Res 2020;12:4747.

58. Yu Y, Shen HM, Fang DM, et al. LncRNA HCP5 promotes the development of cervical cancer by regulating MACC1 via suppression of microRNA-15a. Eur Rev Med Pharmacol Sci 2018;22:4812-9.

59. Ramachandran D, Schürmann P, Mao Q, et al. Association of genomic variants at the human leukocyte antigen locus with cervical cancer risk, HPV status and gene expression levels. Int J Cancer 2020;147:2458-68.

60. Chen J, Zhao D, Meng Q. Knockdown of HCP5 exerts tumor-suppressive functions by up-regulating tumor suppressor miR-128-3p in anaplastic thyroid cancer. Biomed Pharmacother 2019;116:108966.

61. Yuan Q, Fan Y, Liu Z, et al. miR-744-5p mediates lncRNA HOTTIP to regulate the proliferation and apoptosis of papillary thyroid carcinoma cells. Exp Cell Res 2020;392:112024.

62. Chen PY, Li XD, Ma WN, et al. Comprehensive Transcriptomic Analysis and Experimental Validation Identify lncRNA HOXA-AS2/miR-184/COL6A2 as the Critical ceRNA Regulation Involved in Low-Grade Glioma Recurrence. Onco Targets Ther 2020;13:4999-5016.

63. Xiao S, Song B. LncRNA HOXA-AS2 promotes the progression of prostate cancer via targeting miR-509-3p/ PBX3 axis. Biosci Rep 2020;40:BSR20193287.

64. Shi YB, Liu SL, Mou XR, et al. Long noncoding RNA HOXA-AS2 acts as an oncogene by targeting miR-145$3 p$ in human non-small cell lung cancer. Eur Rev Med Pharmacol Sci 2020;24:1243-9. Retracted in: Eur Rev Med Pharmacol Sci 2020;24:8629.

65. Xia F, Chen Y, Jiang B, et al. Long Noncoding RNA HOXA-AS2 Promotes Papillary Thyroid Cancer Progression by Regulating miR-520c-3p/S100A4 Pathway. Cell Physiol Biochem 2018;50:1659-72.

66. Jiang L, Wu Z, Meng X, et al. LncRNA HOXA-AS2 Facilitates Tumorigenesis and Progression of Papillary Thyroid Cancer by Modulating the miR-15a-5p/HOXA3 Axis. Hum Gene Ther 2019;30:618-31.

67. Fan L, Huang X, Chen J, et al. Long Noncoding RNA MALAT1 Contributes to Sorafenib Resistance by Targeting miR-140-5p/Aurora-A Signaling in Hepatocellular Carcinoma. Mol Cancer Ther 2020;19:1197-209.

68. Xu J, Xiao Y, Liu B, et al. Exosomal MALAT1 sponges $\mathrm{miR}-26 \mathrm{a} / 26 \mathrm{~b}$ to promote the invasion and metastasis of colorectal cancer via FUT4 enhanced fucosylation and
Zhao et al. LncRNAs as predictors of prognosis in thyroid cancer

PI3K/Akt pathway. J Exp Clin Cancer Res 2020;39:54.

69. Wu Y, Sarkissyan M, Ogah O, et al. Expression of MALAT1 Promotes Trastuzumab Resistance in HER2 Overexpressing Breast Cancers. Cancers 2020;12:1918.

70. Chu YH, Hardin H, Schneider DF, et al. MicroRNA-21 and long non-coding RNA MALAT1 are overexpressed markers in medullary thyroid carcinoma. Exp Mol Pathol 2017;103:229-36.

71. Samimi H, Haghpanah V, Irani S, et al. Transcript-level regulation of MALAT1-mediated cell cycle and apoptosis genes using dual MEK/Aurora kinase inhibitor "BI-847325" on anaplastic thyroid carcinoma. Daru 2019;27:1-7.

72. Wu X, Li XF, Wu Q, et al. LncRNA SNHG15 predicts poor prognosis in uveal melanoma and its potential pathways. Int J Ophthalmol 2020;13:1195.

73. Long Noncoding RNA SNHG15 Serves as an Oncogene and Predicts Poor Prognosis in Epithelial Ovarian Cancer [Expression of Concern]. Onco Targets Ther 2020;13:6747.

74. Zhang JH, Wei HW, Yang HG. Long noncoding RNA SNHG15, a potential prognostic biomarker for hepatocellular carcinoma. Eur Rev Med Pharmacol Sci 2016;20:1720-4.

75. Xie HW, Wu QQ, Zhu B, et al. Long noncoding RNA SPRY4-IT1 is upregulated in esophageal squamous cell carcinoma and associated with poor prognosis. Tumour Biol 2014;35:7743-54.

76. Pan J, Li XU, Wu W, et al. Long non-coding RNA UCA1 promotes cisplatin/gemcitabine resistance through CREB modulating miR-196a-5p in bladder cancer cells. Cancer Lett 2016;382:64-76.

77. Wang ZQ, He CY, Hu L, et al. Long noncoding RNA UCA1 promotes tumour metastasis by inducing GRK2 degradation in gastric cancer. Cancer Lett 2017;408:10-21.

78. Li Z, Niu H, Qin Q, et al. lncRNA UCA1 mediates resistance to cisplatin by regulating the miR-143/FOSL2signaling pathway in ovarian cancer. Mol Ther Nucleic Acids 2019;17:92-101.

79. Cao Y, Xiong JB, Zhang GY, et al. Long Noncoding RNA UCA1 Regulates PRL-3 Expression by Sponging MicroRNA-495 to Promote the Progression of Gastric Cancer. Mol Ther Nucleic Acids 2020;19:853-64.

80. Li D, Cui C, Chen J, et al. Long non-coding RNA UCA1 promotes papillary thyroid cancer cell proliferation via miR-204-mediated BRD4 activation. Mol Med Rep 2018;18:3059-67.

81. Wang Y, Hou Z, Li D. Long noncoding RNA UCA1 promotes anaplastic thyroid cancer cell proliferation via 
miR-135a-mediated c-myc activation. Mol Med Rep 2018;18:3068-76.

82. Liu H, Li R, Guan L, et al. Knockdown of lncRNA UCA1 inhibits proliferation and invasion of papillary thyroid carcinoma through regulating miR-204/IGFBP5 axis. Onco Targets Ther 2018;11:7197-204.

83. Hombach-Klonisch S, Natarajan S, Thanasupawat T, et al. Mechanisms of therapeutic resistance in cancer (stem) cells with emphasis on thyroid cancer cells. Front Endocrinol (Lausanne) 2014;5:37.

84. Thiery JP, Acloque H, Huang RYJ, et al. Epithelialmesenchymal transitions in development and disease. Cell 2009;139:871-90.

85. Lamouille S, Xu J, Derynck R. Molecular mechanisms of epithelial-mesenchymal transition. Nat Rev Mol Cell Biol 2014;15:178-96.

86. Xu Q, Deng F, Qin Y, et al. Long non-coding RNA regulation of epithelial-mesenchymal transition in cancer metastasis. Cell Death Dis 2016;7:e2254.

87. Dhamija $S$, Diederichs $S$. From junk to master regulators of invasion: lncRNA functions in migration, EMT and metastasis. Int J Cancer 2016;139:269-80.

88. Wang KC, Chang HY. Molecular mechanisms of long noncoding RNAs. Mol Cell 2011;43:904-14.

89. Peng X, Zhang K, Ma L, et al. The Role of Long Non-Coding RNAs in Thyroid Cancer. Front Oncol 2020;10:941.

90. Flockhart RJ, Webster DE, Qu K, et al. BRAFV600E remodels the melanocyte transcriptome and induces BANCR to regulate melanoma cell migration. Genome Res 2012;22:1006-14.

91. Huang Q, Zhang D, Diao Q, et al. lncRNA LINCPINT is downregulated in melanoma and regulates cell proliferation by downregulating lncRNA BANCR. Oncol Lett 2019;18:2917-22.

92. Cai B, Zheng Y, Ma S, et al. BANCR contributes to the growth and invasion of melanoma by functioning as a competing endogenous RNA to upregulate Notch2 expression by sponging miR-204. Int J Oncol 2017;51:1941-51

93. Zhang ZX, Liu ZQ, Jiang B, et al. BRAF activated noncoding RNA (BANCR) promoting gastric cancer cells proliferation via regulation of NF- $\kappa \mathrm{B} 1$. Biochem Biophys Res Commun 2015;465:225-31.

94. Sun M, Liu XH, Wang KM, et al. Downregulation of BRAF activated non-coding RNA is associated with poor prognosis for non-small cell lung cancer and promotes metastasis by affecting epithelial-mesenchymal transition.
Mol Cancer 2014;13:68.

95. Wang D, Wang D, Wang N, et al. Long non-coding RNA BANCR promotes endometrial cancer cell proliferation and invasion by regulating MMP2 and MMP1 via ERK/ MAPK signaling pathway. Cellular physiology and 2016;40:644-56.

96. Liao T, Qu N, Shi RL, et al. BRAF-activated LncRNA functions as a tumor suppressor in papillary thyroid cancer. Oncotarget 2017;8:238-47.

97. Huang G, Wu X, Li S, et al. The long noncoding RNA CASC2 functions as a competing endogenous RNA by sponging miR-18a in colorectal cancer. Sci Rep 2016;6:26524.

98. Xiao XH, He SY. ELF1 activated long non-coding RNA CASC2 inhibits cisplatin resistance of non-small cell lung cancer via the miR-18a/IRF-2 signaling pathway. Eur Rev Med Pharmacol Sci 2020;24:3130-42.

99. Cao Y, Xu R, Xu X, et al. Downregulation of lncRNA CASC2 by microRNA-21 increases the proliferation and migration of renal cell carcinoma cells. Mol Med Rep 2016;14:1019-25

100. Kim CY, Oh JH, Lee JY, et al. The LncRNA HOTAIRM1 Promotes Tamoxifen Resistance by Mediating HOXA1 Expression in ER+ Breast Cancer Cells. J Cancer 2020;11:3416.

101. Chen TJ, Gao F, Yang T, et al. LncRNA HOTAIRM1 Inhibits the Proliferation and Invasion of Lung Adenocarcinoma Cells via the miR-498/WWOX Axis. Cancer Manag Res 2020;12:4379-90.

102. Chao H, Zhang M, Hou H, et al. HOTAIRM1 suppresses cell proliferation and invasion in ovarian cancer through facilitating ARHGAP24 expression by sponging miR106a-5p. Life Sci 2020;243:117296.

103. Coccia EM, Cicala C, Charlesworth A, et al. Regulation and expression of a growth arrest-specific gene (gas5) during growth, differentiation, and development. Mol Cell Biol 1992;12:3514-21.

104. Guo LJ, Zhang S, Gao B, et al. Low expression of long non-coding RNA GAS5 is associated with poor prognosis of patients with thyroid cancer. Exp Mol Pathol 2017;102:500-4.

105.Pellecchia S, Sepe R, Decaussin-Petrucci M, et al. The long non-coding RNA prader willi/angelman region RNA5 (PAR5) is downregulated in anaplastic thyroid carcinomas where it acts as a tumor suppressor by reducing EZH2 activity. Cancers 2020;12:235.

106.Jiang $\mathrm{H}$, Li X, Wang W, et al. Long non-coding RNA SNHG3 promotes breast cancer cell proliferation and 


\section{Page 16 of 16}

metastasis by binding to microRNA-154-3p and activating the notch signaling pathway. BMC Cancer 2020;20:838.

107.Kang R, Yao DF, Xu GZ, et al. The knockdown of SNHG3 inhibits the progression of laryngeal squamous cell carcinoma by miR-340-5p/YAP1 axis and Wnt/ $\beta$-catenin pathway. Neoplasma 2020;67:1094-105.

108. Chen N, Yin D, Lun B, et al. LncRNA GAS8-AS1 suppresses papillary thyroid carcinoma cell growth through the miR-135b-5p/CCND2 axis. Biosci Rep 2019;39:BSR20181440.

109. Gong X, Huang M. Long non-coding RNA MEG3 promotes the proliferation of glioma cells through targeting Wnt/ $\beta$-catenin signal pathway. Cancer Gene Ther 2017;24:381-5.

110.Lubitz CC, Ugras SK, Kazam JJ, et al. Microarray analysis of thyroid nodule fine-needle aspirates accurately classifies benign and malignant lesions. J Mol Diagn 2006;8:490-8.

111. Dai Y, Miao Y, Zhu Q, et al. Expression of long noncoding RNA H19 predicts distant metastasis in minimally invasive follicular thyroid carcinoma. Bioengineered 2019;10:383-9.

112. Wang $\mathrm{Y}$, Lin $\mathrm{X}, \mathrm{Fu} \mathrm{X}$, et al. Long non-coding RNA BANCR regulates cancer stem cell markers in papillary thyroid cancer via the RAF/MEK/ERK signaling pathway. Oncol Rep 2018;40:859-66.

113.Huang F, Zhang Q, Chen W, et al. Long noncoding RNA cancer susceptibility candidate 2 suppresses papillary thyroid carcinoma growth by inactivating the AKT/ERK1/2 signaling pathway. J Cell Biochem 2019;120:10380-90.

Cite this article as: Zhao H, De Souza C, Kumar VE, Nambiar R, Hao D, Zhu X, Luo Y, Liu S, Zhang L, Zhu Z. Long non-coding RNA signatures as predictors of prognosis in thyroid cancer: a narrative review. Ann Transl Med 2021;9(4):359. doi: 10.21037/atm-20-8191

\section{Zhao et al. LncRNAs as predictors of prognosis in thyroid cancer}

114. Shen XH, Qi P, Du X. Long non-coding RNAs in cancer invasion and metastasis. Mod Pathol 2015;28:4-13.

115. Tan J, Liu L, Zuo Z, et al. Overexpression of novel long intergenic non-coding RNA LINC02454 is associated with a poor prognosis in papillary thyroid cancer. Oncol Rep 2020;44:1489-501.

116.Jiao X, Lu J, Huang Y, et al. Long non-coding RNA H19 may be a marker for prediction of prognosis in the follow-up of patients with papillary thyroid cancer. Cancer Biomark 2019;26:203-7.

117. Gao H, Sun X, Wang H, et al. Long noncoding RNA SNHG22 increases ZEB1 expression via competitive binding with microRNA-429 to promote the malignant development of papillary thyroid cancer. Cell Cycle 2020;19:1186-99.

118. Shi T, Gao G, Cao Y. Long Noncoding RNAs as Novel Biomarkers Have a Promising Future in Cancer Diagnostics. Dis Markers 2016;2016:9085195.

119. Gong N, Teng X, Li J, et al. Antisense OligonucleotideConjugated Nanostructure-Targeting lncRNA MALAT1 Inhibits Cancer Metastasis. ACS Appl Mater Interfaces 2019;11:37-42.

120. Ren Y, Wang YF, Zhang J, et al. Targeted design and identification of AC1NOD4Q to block activity of HOTAIR by abrogating the scaffold interaction with EZH2. Clin Epigenetics 2019;11:29.

121. Huarte $M$. The emerging role of lncRNAs in cancer. Nat Med 2015;21:1253-61.

(English Language Editor: R. Scott) 